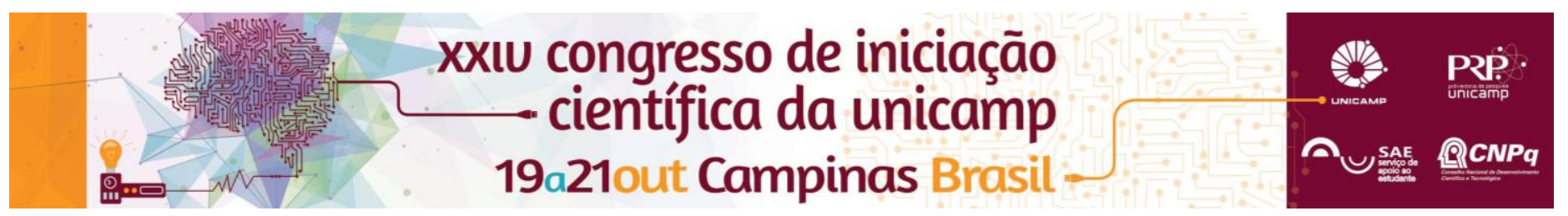

\title{
STROKE: Health Profile Analysis, Nursing Diagnoses and Hospital Care
}

\section{Cássia Milena Freitas Machado Sousa, Ana Railka de Souza Oliveira-Kumakura}

\begin{abstract}
The objective of the study was to investigate in patients affected by stroke the health profile, the nursing diagnoses and hospital care. Descriptive, exploratory, quantitative study in Neurovascular Ambulatory, in a public and tertiary hospital in Campinas. It was investigated socio-demographic data, clinical profile and nursing diagnoses listed of all patients seen in 2015 at the clinic, who made the initial consultation the first follow-up. Data were analyzed using SPSS, version 20.0 under analysis with a descriptive statistical approach. The study was sent to the Research Ethics Committe and approved protocol with 1.163.474. The patients were mostly male, with a mean age of 60 years, with low education levels, people with hypertension, diabetes mellitus, stroke / TIA prior, with sensory, cognitive and motor changes. The degree of disability and dependence, the study revealed that between stroke and outpatient consultation, patients had one decreased autonomy and greater disability. The most listed Nursing diagnoses were those pertecentes the activity / rest area (5) NANDA-I.
\end{abstract}

\section{Key words:}

Stroke ; Health Profile ; Hospitalization

\section{Introduction}

Stoke is responsible for the second cause of death in the world and is the leading cause of physical disability in the developed world..$^{(1,2)}$ In this situation, it is important to know by the multidisciplinary health team on the variables related to the stroke event, to better and more appropriate application of the therapeutic plan established, aiming the well being of the patient. $(2,3)$

This research aimed to investigate the health profile, the nursing diagnoses listed during hospitalization and as was the hospital care of patients who have been affected by stroke and following an outpatient.

\section{Results and Discussion}

The study included 102 patients, mostly male (58.8\%), with civil situation not informed mostly $(57.8 \%)$ and living in Campinas (52\%). The mean age was 60 years.

In the study point out that $65.7 \%$ of the population suffered stroke had Hypertension; $27.5 \%$ of Diabetes Mellitus; 25.5\% stroke / TIA prior, 42.2\% were smoking and reported being active smokers and $21.6 \%$ drank alcohol, while $6.9 \%$ were drug users.

Most patients had a stroke of ischemic type (68.7\%), 49\% the first stroke and $22.5 \%$ agreed with deficit. The signs and symptoms were paresis (40.2\%), dysarthria $(23.5 \%)$, aphasia $(18.6 \%)$, headache $(18.6 \%)$, rhyming deviation (13.7\%), dizziness (10.8\%), hypoesthesia $(10.8 \%)$, right hemiplegia $(9.8 \%)$ decrease in strength $(9.8 \%)$ and weakness (8.8\%).

The degree of disability determined by Rankin Scale at admission highlight asymptomatic (17.6\%) and with symptoms without disabilities (20.6\%); and outpatient they repeated, $23.5 \%$ and $25.5 \%$, respectively. In addition, $13.5 \%$ had moderate to severe disability.

The degree of dependence on the Barthel scale at admission, $26.4 \%$ had a mild dependence, while 1 had total dependence; outpatient consultation, $60.7 \%$ had mild dependence, but $12.8 \%$ had total dependence.

In the study, patients had more, and the three hospital sites (Emergency, Unit Care and Ward) the nursing diagnoses Infection Risk, Self-Care Deficit for Bath, Aspiration Risk and Skin Integrity Risk Impaired.

\section{Conclusions}

The findings of the study show that there was a higher incidence of Ischemic Stroke and the most listed nursing diagnoses are part of the class activity / rest (5) NANDA -I, which directly affect the degree of capacity and dependence of this population.

It is understood that it is important to epidemiological studies that print reliable data and to enable the formulation of public policies for the rehabilitation of these individuals and to prevent new cases since there is an increase in the number of cerebrovascular diseases and chronic non-communicable diseases.

\section{Acknowledgement}

To the team of the Neurovascular Clinic of HC UNICAMP and PIBIC / CNPq, the knowledge opportunity.

1. ROGER, V.L. et al. Heart disease and stroke statistics - 2012 update a report from the American Heart Association. Circulation. v.125:e2-220, 2012;

2. PAULO, RB et al . Acidente vascular cerebral isquêmico em uma enfermaria de neurologia: complicações e tempo de internação. Rev. Assoc. Med. Bras., São Paulo, v. 55, n. 3, p. 313-316, 2009;

3. SANTOS FLSG, et al. Acidente vascular cerebral: o conhecimento dos enfermeiros. Enfermagem em Foco, Santos, 3(2):58-61, 2012. 